\title{
Novel post-Doppler STAP with a priori knowledge information for traffic monitoring applications: basic idea and first results
}

\author{
André B. C. da Silva and Stefan V. Baumgartner \\ Microwaves and Radar Institute, German Aerospace Center (DLR), Oberpfaffenhofen, Germany \\ Correspondence to: André B. C. da Silva (andre.silva@dlr.de)
}

Received: 22 November 2016 - Revised: 4 May 2017 - Accepted: 5 August 2017 - Published: 21 September 2017

\begin{abstract}
This paper presents a novel a priori knowledgebased algorithm for traffic monitoring applications. The powerful post-Doppler space-time adaptive processing (PD STAP) is combined with a known road network obtained from the freely available OpenStreetMap (OSM) database. The road information is applied after the PD STAP for recognizing and rejecting false detections, and moreover, for repositioning the vehicles detected in the vicinity of the roads. The algorithm presents great potential for real-time processing, decreased hardware complexity and low costs compared to state-of-the-art systems. The processor was tested using real multi-channel data acquired by DLR's airborne system F-SAR. The experimental results are shown and discussed, and the novelties are highlighted (e.g., the benefits of using a priori knowledge information).
\end{abstract}

\section{Introduction}

The number of road vehicles is increasing worldwide and the classical methods for monitoring and counting the vehicles on the roads (e.g., stationary sensors like cameras and induction loops) are expensive and limited in spatial coverage. One interesting solution for wide-area traffic monitoring nowadays is the evaluation of geographical coordinates provided continuously via mobile internet connection from the smartphones of cooperative road users.

For traffic monitoring of non-cooperative vehicles (i.e., vehicles not sharing their positions via Internet), Synthetic Aperture Radar (SAR) is also an effective solution due to the wide-area coverage and the independence from daylight and weather conditions (Moreira et al., 2013; Tomiyasu, 1978; Curlander and McDonough, 1991). From the economical point of view, it is clear that generally an airborne radar- based traffic monitoring system cannot compete with a system evaluating continuously the shared geographical positions of cooperative road users. However, especially in case of major events or catastrophes, when the mobile internet connection is disturbed or unavailable and phone communication is impossible, an airborne radar-based system can still provide important information about the actual road traffic situation. This scenario is shown in Fig. 1. Although not discussed in this paper, a radar-based system can additionally provide high resolution SAR images, which can be used for evaluating the condition of the traffic infrastructure (e.g., damage of bridges, landslides over roads). Actual road traffic and traffic infrastructure information is very valuable for relief forces (e.g., fire department, technical emergency service, police), especially for disaster management and evacuation planning.

Numerous ground moving target indication (GMTI) algorithms have been developed for radar-based traffic monitoring applications (Cerutti-Maori et al., 2008; Gierull and Sikaneta, 2003). In particular, a fast dual-channel processor with a priori knowledge information is presented by Baumgartner and Krieger (2012a), where the positions of the vehicles are obtained directly in the intersection between the range-compressed moving target signals and the road axes mapped into the data array. However, it is pointed out that false detections are obtained due to signals coming from adjacent roads. In order to recognize and to discard these false detections, Baumgartner and Krieger (2012b) suggests the use of PD STAP for estimating the direction-of-arrival (DOA) angles of the detections. Although the PD STAP theoretical performance is presented in their paper, the algorithm itself was not implemented and evaluated in detail.

This paper presents an algorithm that combines the powerful PD STAP with a known road network obtained from the 

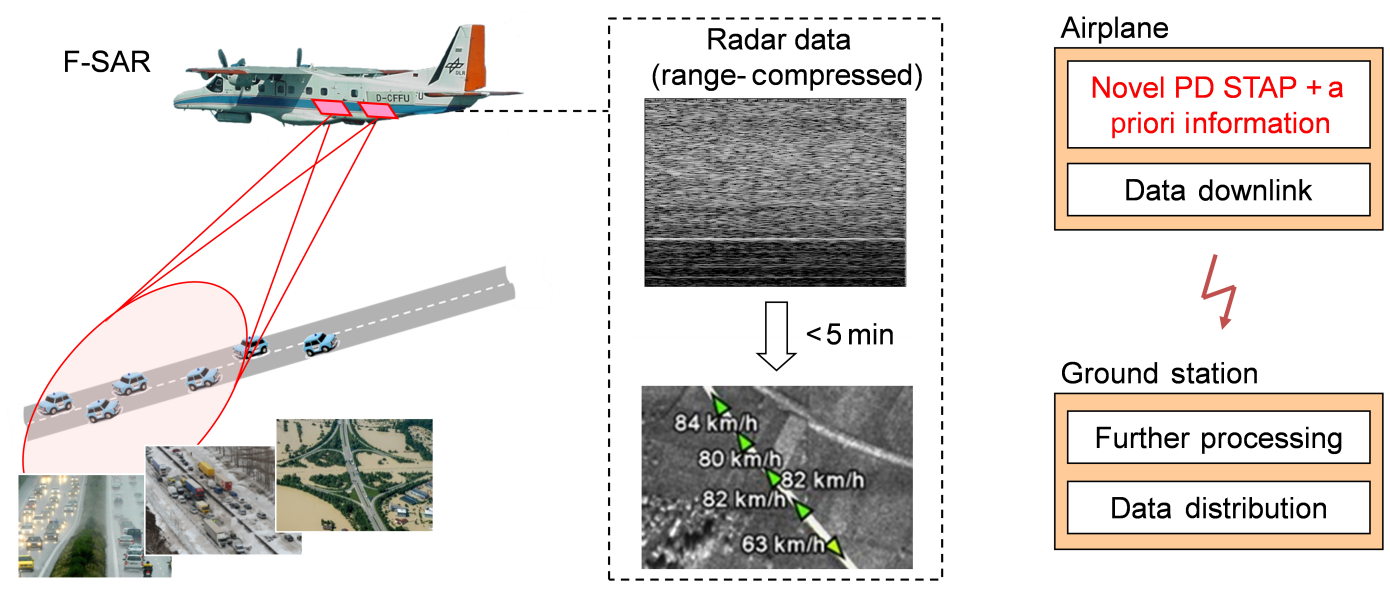

Figure 1. Real-time traffic monitoring in case of catastrophes using SAR and the proposed processor.
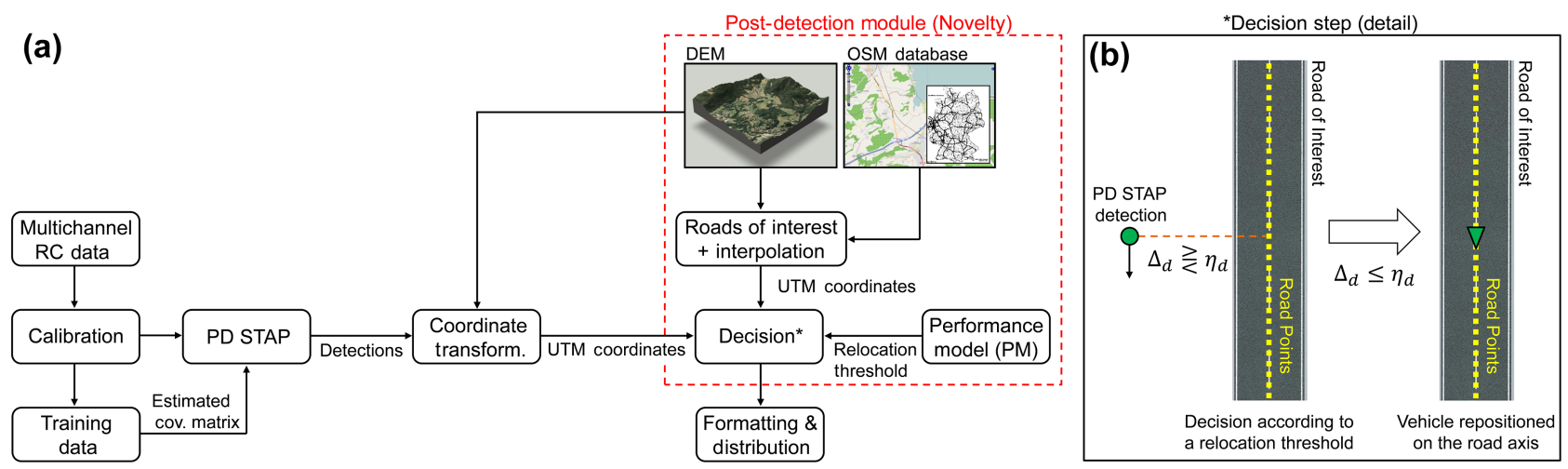

Figure 2. Simplified flowchart of the proposed GMTI algorithm (a) and detail of the decision step (b).

freely available OSM database (OpenStreetMap, 2017). The PD STAP was chosen due to its very good clutter suppression, its sensitivity also to low vehicle velocities, and its accurate target position estimation capabilities (Melvin, 2004; Guerci, 2014; Klemm, 1998). The incorporation of a known road network into the processing chain presents great potential for real-time processing, since only the acquired data related to the roads need to be processed. Thus, decreased processing hardware complexity and low costs compared to the state-of-the-art systems can be achieved. In addition, it is a promising solution for detecting effectively the road vehicles and estimating their positions, velocities and moving directions with high accuracy.

\section{Signal processing algorithm}

The simplified flowchart of the proposed GMTI algorithm is shown in Fig. 2. The processor operates directly on rangecompressed data. After data calibration, the training data are selected in order to estimate the clutter covariance matrix used by the PD STAP for clutter suppression. The PD STAP is well-known (Cerutti-Maori et al., 2010; Ender, 1999) and is used for estimating the line-of-sight velocities and the positions of the targets. The detections are transformed into the Universal Transverse Mercator (UTM) coordinate system, used by the Post-Detection Module.

This module includes two databases: the OSM and a digital elevation model (DEM). The DEM is necessary because the OSM does not provide geographical height information. For each coherent processing interval (CPI), the roads of interest are selected and an interpolation is carried out in order to fill possible gaps between the road points. The OSM provides the geographical positions of the road points, from where it is possible to compute their corresponding road angles with respect to the UTM Easting axis $\left(\alpha_{\mathrm{r}}\right)$ (cf. tangent on road in Fig. 3). The road angle with respect to the flight direction $\left(a_{\mathrm{r}, x}\right)$ allows the computation of the absolute velocity of the target on the road, according to: $v_{\mathrm{t}}=v_{y} / \sin \left(\alpha_{\mathrm{r}, x}\right)$, $\alpha_{\mathrm{r}, x}=\alpha_{\mathrm{r}}-\alpha_{\mathrm{p}}$, where $v_{y}$ is the across-track velocity of the target on ground (computed using the estimated line-of-sight velocity, the DOA angle and the local incidence angle) and $\alpha_{\mathrm{p}}$ is the platform heading angle with respect to the UTM Easting axis, as shown in Fig. 3. 


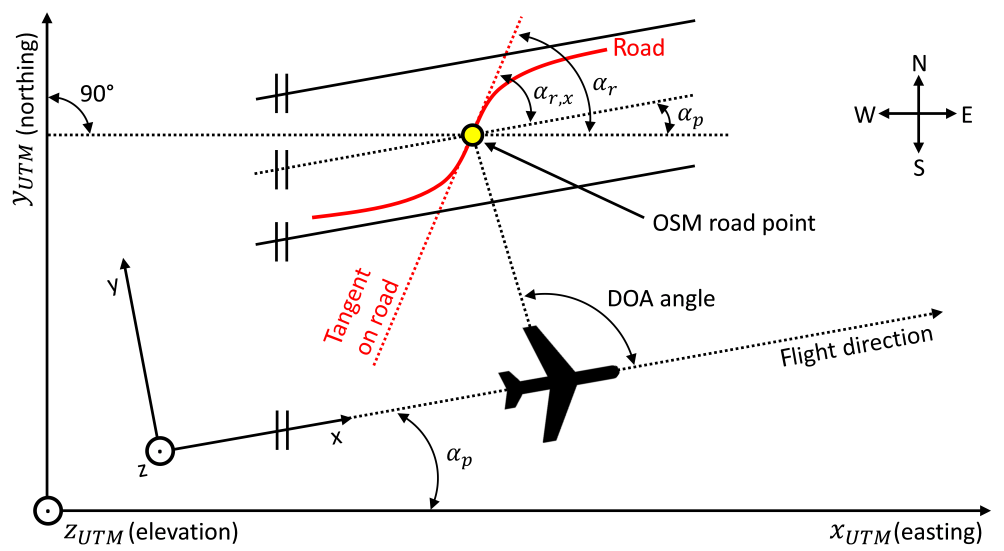

Figure 3. Relation between the geographical Cartesian UTM coordinate system $\left\{x_{\mathrm{UTM}}, y_{\mathrm{UTM}}, z_{\mathrm{UTM}}\right\}$ and local Cartesian coordinate system $\{x, y, z\}$. The figure was modified after Baumgartner and Krieger (2012a).
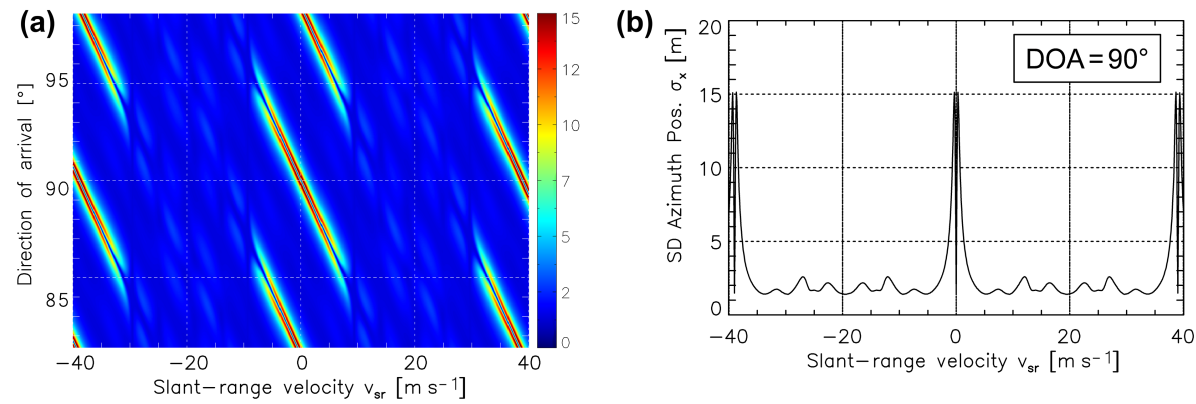

Figure 4. Standard deviation of the azimuth relocation error for one particular detection at the PD STAP output. Wide range of DOA angles (a) and a cut along the broadside direction (i.e., $\left.\mathrm{DOA}=90^{\circ}\right)(\mathbf{b})$.

In the Decision step (cf. Fig. 2b), the distance $\Delta_{\mathrm{d}}$ between the estimated position of the target and its closest road point is measured and compared to a relocation threshold $\eta_{\mathrm{d}}$ in order to decide whether the target corresponds to a true road vehicle or to a false detection. If the first condition is fulfilled, the target is repositioned to the closest road point; otherwise it is discarded. The relocation threshold is computed adaptively for each detection by using an appropriate Performance Model (cf. Sect. 3). Finally, the data are formatted and distributed, e.g., to the traffic management center.

\section{Performance model}

The PD STAP performance model uses the framework introduced in Ender et al. (2008) and the system parameters given in Baumgartner and Krieger (2012b). The signal-toclutter plus noise ratio (SCNR) and the line-of-sight velocity of each PD STAP detection are used as input parameters, so that an adaptive relocation threshold can be computed. For instance, Fig. 4 shows the expected azimuth relocation error for one particular detection with a signal-to-noise ratio (SNR) of $25 \mathrm{~dB}$, where the maximum relocation error at the broadside direction is around $15 \mathrm{~m}$ (cf. Fig. $4 \mathrm{~b}$ ). The clutterto-noise ratio (CNR) is $33 \mathrm{~dB}$ and the PRF is $2500 \mathrm{~Hz}$. It has to be pointed out that this is an optimal case, since additional errors may arise (e.g., due to the pointing direction and the road axis uncertainties - in the order of 5-10 $\mathrm{m}$ for the OSM). The additional errors will be considered in the next version of the proposed algorithm.

\section{Experimental data}

The proposed algorithm was tested using real 4-channel aperture switching data acquired by DLR's airborne system FSAR. The flight campaign was conducted in February 2007 over the Allgäu airport in Memmingen, where five controlled cars were considered (Baumgartner et al., 2007). The velocities of the vehicles and the radar parameters are given in Baumgartner and Krieger (2012a). The data were processed using CPIs of $1024 \times 128$ range-Doppler samples, and the beamformers were applied using DOA angle steps of $0.1^{\circ}$ within an interval determined by the azimuth antenna beam width. 


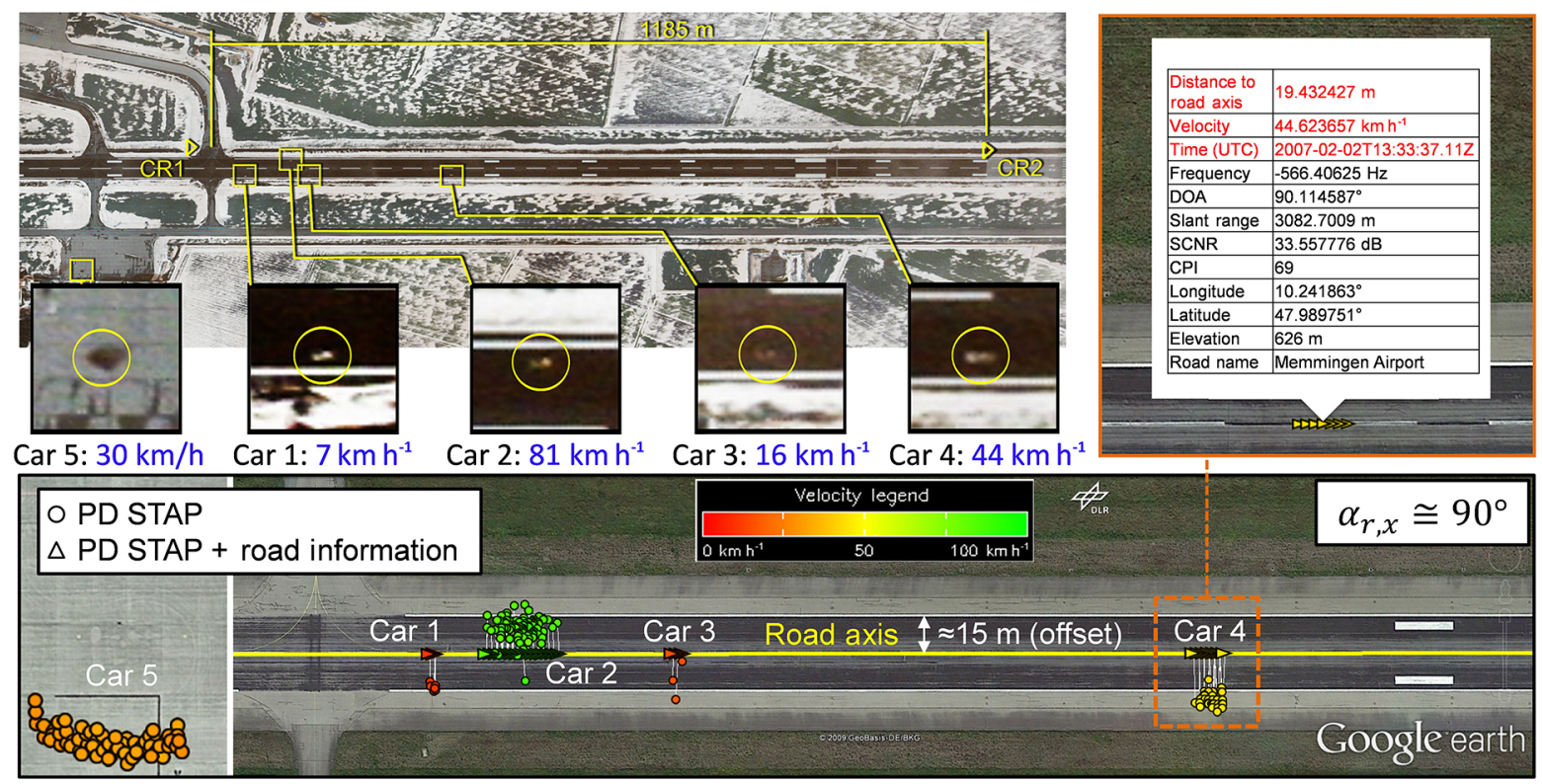

Figure 5. Results from the flight campaign over the Allgäu airport in Memmingen. Optical reference data (top left); Google Earth images overlaid with radar detections before (circle) and after (triangle) reposition using a priori knowledge information (bottom); detail of Car 4 showing the estimated parameters at the time instant 13:33:37.11 UTC (top right). The road axis is shown in yellow.

The experimental results are shown in Fig. 5. The optical reference data (acquired simultaneously with the radar data) are shown at the top left, where the cars $1-4$ move on the edges of the runway and Car 5 moves "off-road" in circle. The radar detections are shown at the bottom as a Google Earth overlay. The detections are shown before (circles) and after (triangles) reposition using the road information, and the center of the runway (yellow line) was considered as the road axis. The colors are related to the absolute velocities of the cars, the triangles point to their moving direction, and the thin white lines connect the PD STAP detections to their closest road points (on the road axis).

It is pointed out that an offset of around $15 \mathrm{~m}$ is introduced, since the center of the runway is used as the road axis and the cars moved on the edges (the width of the conventional road lanes is in the order of $3.5 \mathrm{~m}$ and therefore the offset to the road axis is much smaller in real road data scenarios). The detections of Car 4 are shown in detail at the top right. The estimated absolute velocity of Car 4 is $44.62 \mathrm{kmh}^{-1}$ at the time instant 13:33:37.11 UTC, which agrees with the differential GPS reference data (Baumgartner and Krieger, 2012a). In addition, its estimated position accuracy is better than $5 \mathrm{~m}$ taking into account the road axis offset of $15 \mathrm{~m}$. Car 5 was rejected after applying the road information because it moves "off-road".

Finally, note that the PD STAP detected the cars several times due to the small CPIs used (the CPIs are much smaller than the overall illumination time determined by the width of the azimuth antenna pattern). Thus, a tracking algorithm in a post processing step would be helpful to assign multiple detections of the same car to one physical object. However, the tracking algorithms are beyond the scope of this paper.

Figure 6 shows two velocity histograms of the detections obtained from the Memmingen's experiment, assuming a relocation threshold of $\eta_{\mathrm{d}}=20 \mathrm{~m}$. In Fig. 6a, the absolute ground range velocities $\left|v_{y}\right|$ of all PD STAP detections are shown before applying the road information and the relocation threshold. Note that the cars 1 and 3 cannot be clearly identified due to numerous false detections. In Fig. 6b, after applying the road information and discarding potential false detections (i.e., detections farther than $20 \mathrm{~m}$ from the road axis), the absolute velocities on the road $\left|v_{t}\right|$ of the remaining detections are shown. The result is a clear histogram where the cars 1-4 can be easily identified (with an estimated SCNR of $20-35 \mathrm{~dB}$ ).

It is important to mention that the comparison between both histograms shown in Fig. 6 could be done especially because the cars moved nearly in range direction (i.e., $\alpha_{\mathrm{r}, x} \cong$ $90^{\circ}$ ) and therefore their ground range velocities (i.e., lineof-sight velocities projected on ground) were nearly equal to their absolute velocities on the road.

Finally, the proposed processor was tested using a data take containing real autobahn traffic scenario. The flight campaign was conducted in October 2013 over the Greifenberg area. Figure 7 shows the experimental results, where numerous cars are detected on the highway A96 and on residential roads. The considered roads from the OSM database are shown in white. Since no ground truth data were available in this experiment, it is not possible to determine the probability of detection, the false alarm rate and the errors of the 

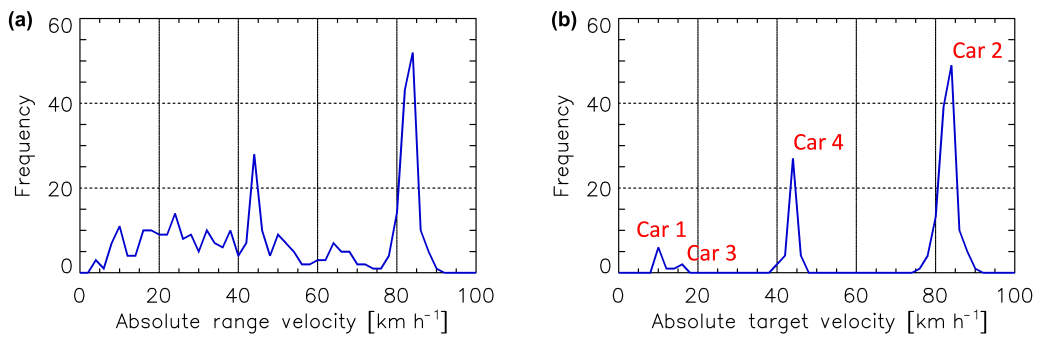

Figure 6. Velocity histograms of the detections from Memmingen's experiment: absolute ground range velocities $\left|v_{y}\right|$ of all PD STAP detections (a); absolute velocities on the road $\left|v_{t}\right|$ of the remaining detections after applying the road information (b).

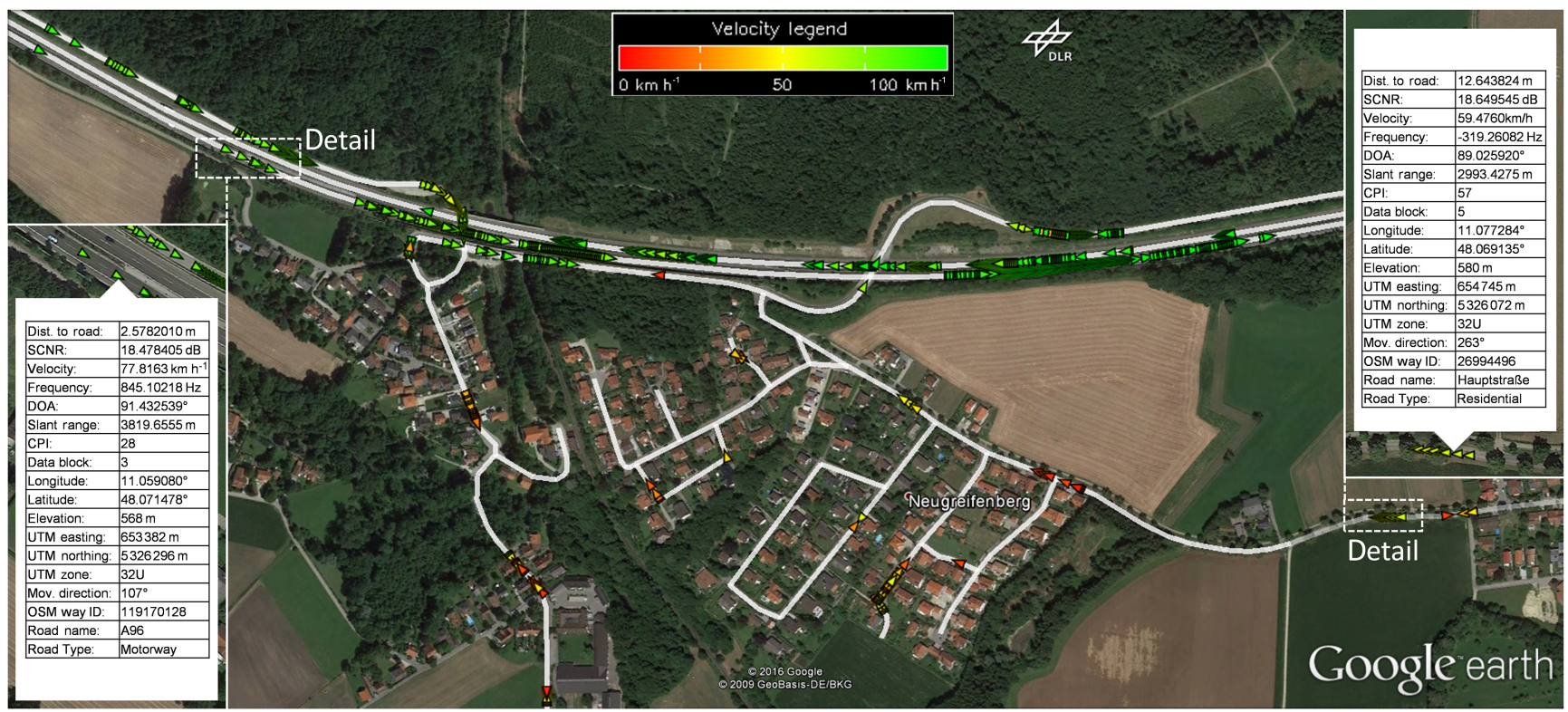

Figure 7. Results from the flight campaign over the Greifenberg area. Google Earth image overlaid with radar detections and their corresponding velocity estimations after using the road information. The cars (triangles) were automatically detected and their parameters were estimated using the proposed processor. The considered roads from the OSM database are shown in white.

estimated parameters. Nevertheless, the estimated velocities on the highway A96 and on the residential roads are reasonable.

\section{Conclusion}

The experimental results revealed a powerful GMTI algorithm that detects even slow vehicles and discards many false detections, being suitable for many traffic monitoring applications. In the Memmingen's experiment, the proposed processor detected vehicles as slow as $7 \mathrm{kmh}^{-1}$ with an overall position estimation accuracy limited by the relocation threshold. The estimated velocities of the vehicles were in very good agreement with the differential GPS reference data. Although no ground truth data were available in the Greifenberg's experiment, the estimated velocities of the detected vehicles are reasonable. Before the algorithm can be used operationally, a clustering and tracking algorithm still needs to be developed.
Data availability. The data used within this research project were acquired by DLR's F-SAR airborne radar sensor achieving resolution in the sub-meter range. Therefore, all data products are either restricted to DLR internal evaluation/analyses or subject to export control regulations. In this context, especially the SAR raw data, on which this research is based on, are excluded for public accessibility.

Competing interests. The authors declare that they have no conflict of interest.

Special issue statement. This article is part of the special issue "Kleinheubacher Berichte 2016". It is a result of the Kleinheubacher Tagung 2016, Miltenberg, Germany, 26-28 September 2016. 
Acknowledgements. This work was supported by grants from the German Academic Exchange Service (DAAD). The authors are grateful to the reviewers whose comments and suggestions significantly improved both clarity and precision of this paper.

Edited by: Jens Anders

Reviewed by: two anonymous referees

\section{References}

Baumgartner, S., Gabele, M., Gebert, N., Scheiber, R., Krieger, G., Bethke, K.-H., and Moreira, A.: Digital beamforming and traffic monitoring using the new FSAR system of DLR, Proceedings of International Radar Symposium (IRS), Cologne, Germany, 5-7 September 2007, 5 pp., 2007.

Baumgartner, S. V. and Krieger, G.: Fast GMTI algorithm for traffic monitoring based on a priori knowledge, IEEE T. Geosci. Remote, 50, 4626-4641, 2012a.

Baumgartner, S. V. and Krieger, G.: A priori knowledge-based Post-Doppler STAP for traffic monitoring applications, 2012 IEEE International Geoscience and Remote Sensing Symposium (IGARSS), Munich, Germany, 22-27 July 2012, 6087-6090, 2012b.

Cerutti-Maori, D., Klare, J., Brenner, A. R., and Ender, J. H. G.: Wide-area traffic monitoring with the SAR/GMTI system PAMIR, IEEE T. Geosci. Remote, 46, 3019-3030, 2008.

Cerutti-Maori, D., Gierull, C. H., and Ender, J. H. G.: Experimental verification of SAR-GMTI improvement through antenna switching, IEEE T. Geosci. Remote, 48, 2066-2075, 2010.
Curlander, J. C. and McDonough, R. N.: Synthetic aperture radar: systems and signal processing, Wiley, New York, 1991.

Ender, J. H. G.: Space-time processing for multichannel synthetic aperture radar, Electron. Commun. Eng., 11, 29-38, 1999.

Ender, J. H. G., Gierull, C. H., and Cerutti-Maori, D.: Improved space-based moving target indication via alternate transmission and receiver switching, IEEE T. Geosci. Remote, 46, 3960-3974, 2008.

Gierull, C. H. and Sikaneta, I. C.: Raw data based two-aperture SAR ground moving target indication, Geoscience and Remote Sensing Symposium, 2003, IGARSS '03, Proceedings, 2003 IEEE International, Toulouse, France, 21-25 July 2003, 2, 1032-1034, 2003.

Guerci, J. R.: Space-time adaptive processing for radar, Artech House, 2014.

Klemm, R.: Space-time adaptive processing, The Institute of Electrical Engineers, London, UK, 1998.

Melvin, W. L.: A STAP overview, IEEE Aerospace and Electronic Systems Magazine, 19, 19-35, 2004.

Moreira, A., Prats-Iraola, P., Younis, M., Krieger, G., Hajnsek, I., and Papathanassiou, K. P.: A tutorial on synthetic aperture radar, IEEE Geoscience and Remote Sensing Magazine, 1, 6-43, 2013.

OpenStreetMap - The Free Wiki World Map, OpenStreetMap Foundation (OSMF), available at: http://www.openstreetmap. org, last access: January 2017.

Tomiyasu, K.: Tutorial review of synthetic-aperture radar (SAR) with applications to imaging of the ocean surface, Proceedings of the IEEE, 66, 563-583, 1978. 